\title{
Des greffes dans le cerveau
}

Plusieurs centaines de patients atteints de maladie de Parkinson ont reçu des greffes intracérébrales depuis avril 1987. C'est en effet dans le numéro du New England Journal of Medicine du début avril 1987 qu'une équipe de neurochirurgiens mexicains a rapporté les deux premiers succès thérapeutiques obtenus grâce à la réalisation de greffes intracérébrales. Mais l'attente et la préparation de très nombreuses équipes neurochirurgicales étaient telles que, en un an, c'est plusieurs dizaines d'équipes qui, dans de très nombreux pays quoique pas encore en France, au moment où nous écrivons - se sont lancées dans cette aventure. Il

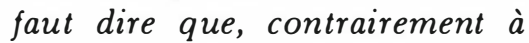
d'autres greffes d'organes, il ne s'agit pas ici d'un exploit technique ou d'une performance artistique des chirurgiens.

La maladie de Parkinson est liée à la dégénérescence des neurones du locus niger qui produisent de la dopamine. Les troubles apparaissent lorsque la concentration en dopamine s'abaisse en dessous d'un seuil déterminé dans le striatum. La dopamine est un des maillons de la chaîne des catécholamines qui donne naissance à la noradrénaline et à l'adrénaline. On a donc pensé dès les premières greffes intracérébrales chez le rat à utiliser les cellules chromaffines de la médullo-surrénale pour pallier la faillite des neurones dopaminergiques. La quasi-totalité des greffes réalisées chez les malades parkinsoniens s'appuie sur cette idée. Une équipe de chirurgiens prélève chez le patient une des glandes surrénales et, dans le même temps, une équipe de neurochirurgiens réimplante la médullo-surrénale découpée en morceaux au niveau d'un striatum, introduisant ainsi dans le cerveau une petite usine à catécholamines. Cette double intervention nécessite certes une bonne coordination entre deux équipes de chirurgiens rarement appelés à collaborer, mais elle ne présente de difficultés que parce que les malades sont atteints d'une rigidité musculaire créant quelques problèmes, notamment respiratoires. En raison de ces difficultés seuls des malades jeunes (moins de 60 ans) ont été opérés jusqu'à présent, ce qui limite l'expérience à une population assez particulière de malades puisque la maladie de Parkinson apparaît, le plus souvent, chez la personne âgée. Les fondements théoriques de cette intervention sont cependant plus flous que le simple rappel de la chaîne des catécholamines peut le laisser penser car, a priori, les cellules chromaffines implantées dans le cerveau produisent de la noradrénaline, ainsi que de nombreux peptides, mais très peu de dopamine (voir m/s no 10 , vol. 3, p. 614). Pourtant, et quelles qu'en soient les causes exactes, l'interiention semble entraîner une nette amélioration clinique chez plus d'un malade sur trois. On attend donc avec beaucoup d'intérêt les premières confrontations entre les différentes équipes réalisant ce genre d'interventions et les premiers bilans chiffrés concernant tant les succès et échecs thérapeutiques que leur éventuelle association avec certaines données d'examens biologiques ou radiologiques. Un congrès mondial est prévu pour réaliser ce premier bilan en août 1989.

Les chirurgiens mexicains ont annoncé en janvier 1988 la réalisation d'une première greffe de neurones embryonnaires dopaminergiques (provenant de foetus humains) chez un patient parkinsonien. Une équipe suédoise a également pratiqué deux interventions équivalentes. Ce type de transplantations est différent $d u$ précédent car il s'agit-là non plus d'auto- mais d'hétérogreffes avec donneurs, ce qui pose bien sûr les problèmes d'histocompatibilité. Le cerveau semble, de ce point de vue, un organe privilégié puisqu'il est possible dans certaines conditions d'y réaliser - chez le rat - des xénogreffes (greffes entre espèces) et cela sans immunosuppression. Ces greffes de neurones embryonnaires ouvrent sans aucun doute des perspectives bien plus grandes que les autogreffes de cellules chromaffines car d'une part la variété des neurones " greffables » est très vaste et d'autre part leur intégration et leur développement dans le cerveau-hôte sont apparement excellents. On envisage ainsi dans l'avenir des greffes spécifiques de neurones striataux chez des malades porteurs d'une chorée de Huntington, de neurones cholinergiques dans le cortex cérébral des patients atteints de maladie d'Alzheimer, etc. Bref, les maladies neurodégénératives semblent enfin à la portée de la thérapeutique.

M. Peschanski

FLASH : Un moratoire sur les transplantations intracérébrales de tissu médullosurrénalien vient d'être publiquement accepté, lors d'un Congrès International à Jérusalem, par les principales équipes de neurochirurgiens qui pratiquaient cette intervention, y compris l'équipe mexicaine d'Ignazio Madrazo, pionnière de l'expérience. Les doutes quant au bien-fondé de l'intervention et les réserves quant à son efficacité réelle ont donc prévalu, jusqu'à l'année prochaine puisque le bilan de l'opération sera réalisé en août 89 au Congrès de Lund et que le moratoire sera alors réévalué. Le temps, peut-être, pour les recherches expérimentales - notamment chez le primate - d'apporter quelques réponses. 